\title{
Importance of three-dimensional grids and time-dependent factors for applications of earthquake forecasting models to subduction environments
}

\author{
Chung-Han Chan \\ Earth Observatory of Singapore, Nanyang Technological University, Singapore \\ Correspondence to: Chung-Han Chan (hantijun@googlemail.com)
}

Received: 20 October 2015 - Published in Nat. Hazards Earth Syst. Sci. Discuss.: 18 December 2015

Revised: 26 July 2016 - Accepted: 5 September 2016 - Published: 28 September 2016

\begin{abstract}
This study provides some new insights into earthquake forecasting models that are applied to regions with subduction systems, including the depth component for forecasting grids and time-dependent factors. To demonstrate the importance of depth component, a forecasting approach, which incorporates three-dimensional grids, is compared with an approach with two-dimensional cells. Through application to the two subduction regions, Ryukyu and Kanto, it is shown that the approaches with three-dimensional grids always demonstrate a better forecasting ability. I thus confirm the importance of depth dependency for forecasting, especially for applications to a subduction environment or a region with non-vertical seismogenic structures. In addition, this study discusses the role of time-dependent factors for forecasting models and concludes that time dependency only becomes crucial during the period with significant seismicity rate change that follows a large earthquake.
\end{abstract}

\section{Introduction}

Earthquake forecasting models generally provide essential knowledge for seismic hazards mitigation; i.e. they point out the regions with high seismicity activity and provide fundamental information regarding seismic hazard assessment (Marzocchi et al., 2003; Lombardi and Marzocchi, 2009). Therefore, studies and general interest in this issue have significantly increased and many forecasting models have been proposed.

However, most forecasting studies focus on the crustal earthquakes; i.e. the credibility of these models remains con- troversial for the application to subduction environments. Such regions include non-vertical seismogenic structures; depth-independent grid cells thus become crucial for forecasting models. Besides spatial distribution, temporal evolution of seismicity is another factor that dominates forecasting precision. Wiens et al. (1997) concluded that in comparison to the sequence of crustal earthquakes, a smaller number of aftershocks follows the occurrence of a subduction event. Such differences in temporal behaviour might result in a forecasting bias.

Therefore, this study applies several forecasting models and discusses their feasibility for applications to subduction regions. To model the behaviours of non-vertical seismogenic structures precisely, approaches with threedimensional grids cells are developed. By comparing these approaches with those with two-dimensional cells, I show the importance of the depth component. To reveal the role of the temporal factor for forecasting, I evaluate the forecasting ability of time-independent and renewal models. I apply the models to two subduction regions, the southwestern portion of the Ryukyu and Kanto.

\section{Forecasting models}

To examine the factors that control the feasibility of forecasting models in a subduction environment, this study introduces two forecasting approaches, the smoothing kernel function and the rate-and-state friction model, described below. 


\subsection{The smoothing kernel function}

Woo (1996) proposed a forecasting model, which described time-independent seismicity rate density $\lambda(M, x)$ at the site of interest, $x$, as a function of magnitude, $M$, as follows:

$\lambda(M, x)=\sum_{i=1}^{N_{\mathrm{M}}} \frac{K\left(M, \overline{x-x_{i}}\right)}{T_{\mathrm{M}}}$

where $K\left(M, \overline{x-x_{i}}\right)$ represents a smoothing kernel as a function of magnitude and distance between the site of interest, $x$, and the location of the $i$ th earthquake, $x ; T_{\mathrm{M}}$ represents the period of a complete catalogue with a magnitude threshold; and $N_{\mathrm{M}}$ represents the total number of earthquakes with magnitudes larger than the threshold. The unit for $\lambda(M, x)$ can be per area or volume, depending on the dimensions of forecasting models. Although $\lambda(M, x)$ is a function of magnitude, for simple representation, forecasting seismicity rates in different magnitude ranges will be shown in this study. This study follows the procedure of Woo (1996) and describes the kernel function $K\left(M, \overline{x-x_{i}}\right)$ as follows:

$K\left(M, \overline{x-x_{i}}\right)=\frac{\mathrm{PL}-1}{\pi H^{2}(M)}\left(1+\left(\frac{\overline{x-x_{i}}}{H(M)}\right)^{2}\right)^{-\mathrm{PL}}$,

where PL represents the power law index and $H(M)$ represents the bandwidth function, defined as the nearest distance to other events for each magnitude bin, $M$. The function can be represented as follows:

$H(M)=c \cdot e^{d \cdot M}$,

where $c$ and $d$ are constants and $c$ is a length, obtained from regression analysis of earthquake spatial distribution. Molina et al. (2001) recommend a PL of between 1.5 and 2.0, and Chan et al. (2010) concluded insignificant differences between the results when the PL is assumed to be between the recommended values. This study thus assumed an intermediate value of 1.75 . The kernel function represents seismicity rate as a function of magnitude, and its feasibility has been proven through implementation in various regions (e.g. Molina et al., 2001; Beauval et al., 2006; Chan et al., 2010, 2012).

The smoothing kernel function forecasts seismicity rate based on the seismic activity during an observation period; i.e. this model minimizes the factor of temporal evolution and provides a time-independent model.

\subsection{The rate-and-state friction model}

Another implemented forecasting approach is the rate-andstate friction model (Dieterich, 1994), which evaluates seismicity rate evolution based on earthquake Coulomb stress changes. According to the constant apparent friction law (Harris, 1998; Cocco and Rice, 2002), Coulomb stress change by a source event, $n, \Delta \mathrm{CFS}_{n}(x)$ at the site of interest $x$ as a function of magnitude, $M$, and time, $t$, as below:

$\Delta \operatorname{CFS}_{n}(x)=\Delta \tau_{n}(x)+\mu^{\prime} \Delta \sigma_{n}(x)$,

where $\Delta \tau_{n}(x)$ represents the shear stress change along the slip direction; $\mu^{\prime}$ represents the apparent friction coefficient; and $\Delta \sigma_{n}(x)$ represents the normal stress change on the assumed plane. The law suggests that a positive Coulomb stress change enhances the occurrence of subsequent events, while a negative one inhibits future seismic activity. According to the law, however, the Coulomb stress change model does not quantify seismicity rate changes.

To quantify seismicity rate evolution, Dieterich (1994) proposed the rate-and-state friction model. This model presents the evolution of the seismicity rate $\Delta R_{n}(M, x, t)$ by considering the Coulomb stress change by the $n$th source event $\Delta \mathrm{CFS}_{n}(x)$ as below:

$$
\begin{aligned}
& \Delta R_{n}(M, x, t) \\
& =\frac{\lambda(M, x)}{\left[\frac{\lambda(M, x)}{\Delta R_{n-1}(M, x)} \exp \left(-\frac{\Delta \mathrm{CFS}_{n}(x)}{A \sigma}\right)-1\right] \exp \left(-\frac{t-t_{n}}{t_{\mathrm{a}}}\right)+1},
\end{aligned}
$$

where $\lambda(M, x)$ represents the time-independent seismicity rate shown in Eq. (1); $\Delta R_{n-1}(M, x)$ represents the seismicity rate change just before the occurrence of the $n$th source event (i.e., $\left.\Delta R_{0}=\lambda(M, x)\right)$; $A \sigma$ represents a constitutive parameter of the model with the dimension of a stress; $t_{n}$ represents the occurrence time of the $n$th source event; and $t_{\mathrm{a}}$ represents the aftershock duration. The rate-and-state friction model forecasts the temporal evolution of seismicity rate after occurrence of large earthquakes.

\section{Forecasting application to the Ryukyu region}

\subsection{Tectonic setting and earthquake catalogue}

The southwestern portion of the Ryukyu trench near Taiwan is seismically active as the Philippine Sea Plate subducts under the Eurasian Plate from the south (Fig. 1). In addition to high seismic activity, this region contains a good-quality earthquake catalogue. The Taiwan Telemetered Seismic Network (TTSN), the modern seismic network in the Taiwan region, was initiated in the early 1970s (Tsai et al., 1981). Since its operation, approximately 4000 earthquake events have been recorded each year. After the early 1990s, TTSN stations were integrated into the Central Weather Bureau Seismic Network (CWBSN), which records approximately 20000 events each year (Shin, 1992). With a large amount of seismic activity and high-quality earthquake catalogues, the region is an ideal site for earthquake forecasting tests. 
Table 1. Source parameters for the source events used for the inputs of the rate-and-state friction model. Earthquakes with $M_{\mathrm{W}} \geq 4.5$ that occurred in 2010 and 2011 were considered. Parameters were determined based on the Broadband Array in Taiwan for Seismology (BATS).

\begin{tabular}{ccccccccccr}
\hline No. of event & Year & Month & Day & Longitude & Latitude & Depth $(\mathrm{km})$ & Magnitude & Strike & Dip & Rake \\
\hline 1 & 2010 & 2 & 26 & 122.84 & 23.60 & 44 & 5.0 & 200.9 & 33.8 & 97.7 \\
2 & 2010 & 6 & 15 & 121.63 & 24.06 & 16 & 5.1 & 261.3 & 42.8 & 142.5 \\
3 & 2010 & 7 & 8 & 122.00 & 24.40 & 24 & 4.7 & 290.6 & 21.4 & -110.9 \\
4 & 2010 & 7 & 9 & 122.66 & 24.66 & 116 & 4.8 & 216.5 & 60.9 & 20.4 \\
5 & 2010 & 8 & 30 & 122.11 & 24.92 & 11 & 4.6 & 189.8 & 26.5 & -141.1 \\
6 & 2010 & 11 & 12 & 122.43 & 24.05 & 29 & 4.6 & 327.7 & 65.9 & 160.4 \\
7 & 2010 & 11 & 21 & 121.75 & 23.83 & 46 & 5.2 & 248.9 & 22.4 & 141.3 \\
8 & 2011 & 2 & 1 & 121.80 & 24.24 & 23 & 4.9 & 329.1 & 27.7 & -131.0 \\
9 & 2011 & 5 & 22 & 121.72 & 24.15 & 19 & 4.7 & 215.1 & 63.9 & -4.3 \\
\hline
\end{tabular}

\subsection{Procedure of application}

\subsubsection{The smoothing kernel function}

Implementing a complete portion of an earthquake catalogue is a key factor for precise forecasts. I checked the magnitude of completeness, $M_{\mathrm{c}}$, for the catalogues using the maximum curvature approach (Wiemer and Wyss, 2000). Due to station coverage, both the TTSN and CWBSN catalogues (represented in Fig. 1a and b, respectively) obtain better observation quality inland than in the offshore region. The $M_{\mathrm{c}}$ for the CWBSN catalogue (Fig. 1b) was lower than that for the TTSN (Fig. 1a), and the regions with a $M_{\mathrm{c}} \leq 4.0$ for the TTSN and a $M_{\mathrm{c}} \leq 3.0$ for the CWBSN are nearly the same. Thus, the intersection of the two catalogues, regions with $M_{\mathrm{c}} \leq 4.0$ for TTSN and $M_{\mathrm{c}} \leq 3.0$ for the CWBSN (Fig. 1c), determines the study region and the magnitude thresholds. I applied the earthquakes before 2009 for model construction and referred those in 2010 and 2011 as forecasting events for retrospective tests. Thus, $T_{\mathrm{M}}$ is 37 and 14 years, while $N_{\mathrm{M}}$ is the total number of earthquakes with magnitudes larger than 4.0 and 3.0 for TTSN and CWBSN cases, respectively. Based on the parameters of the earthquakes before 2009, the linear regression determined that the $c$ and $d$ values of the bandwidth function in Eq. (3) were $0.0174 \mathrm{~km}$ and 1.1209 , respectively.

\subsubsection{The rate-and-state friction model}

To calculate Coulomb stress change $(\triangle \mathrm{CFS})$, rupture behaviours of source earthquakes and source parameters of receiver fault planes are two important factors. For the source earthquake parameters, the hypocentre location, the moment magnitude and the focal mechanisms were obtained from the Broadband Array in Taiwan for Seismology (BATS) website (http://bats.earth.sinica.edu.tw/), and fault dimension and magnitude of slip were determined through the scaling laws of Yen and Ma (2011). For source parameters of receiver fault planes, both location and focal mechanism should be defined and utilized for the calculation. The loca-

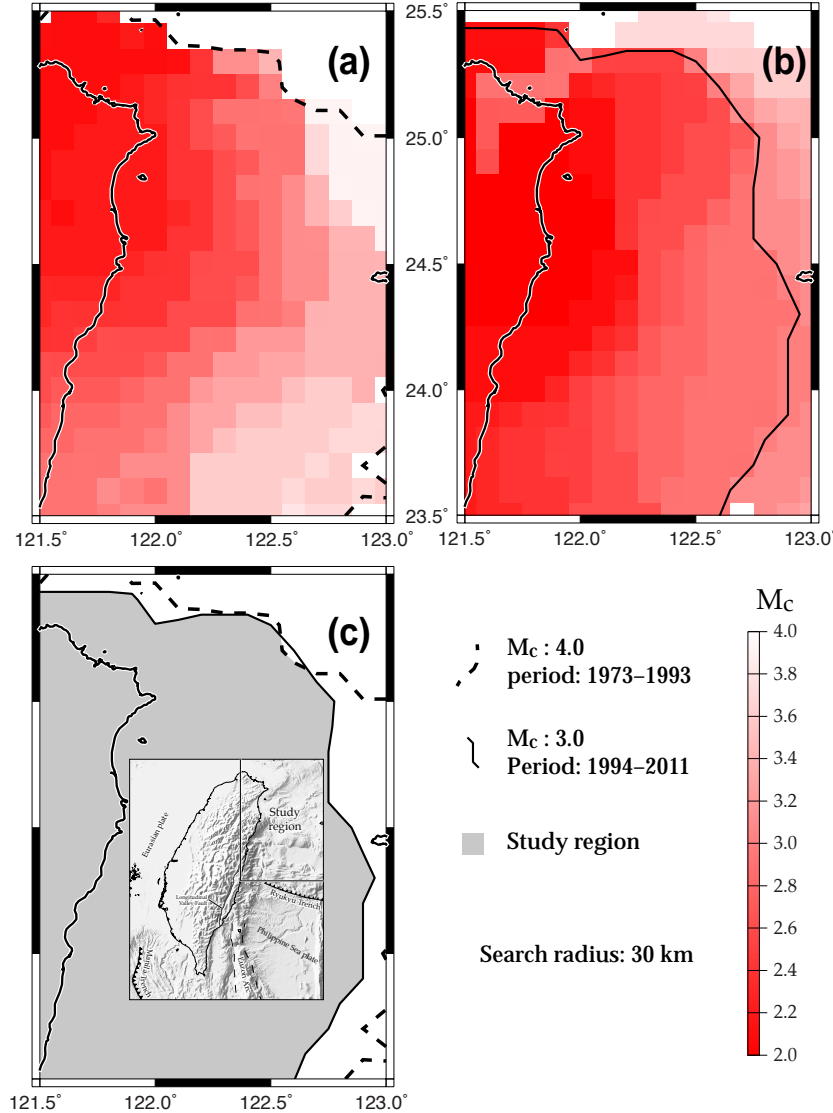

Figure 1. The magnitude of completeness $\left(M_{\mathrm{c}}\right)$ for (a) the TTSN and (b) the CWBSN catalogues. (c) The study area is shown in grey, with the intersection of regions with $M_{\mathrm{c}} \leq 4.0$ for the TTSN shown with dashed lines and with $M_{\mathrm{c}} \leq 3.0$ for the CWBSN shown with solid lines. The $M_{\mathrm{c}}$ for each grid is determined according to the events that occurred within $30 \mathrm{~km}$ of the centre of each grid.

tions are assumed to be the coordinates and the target depth of each calculation grid. For receiver fault mechanisms, I followed the procedure of Catalli and Chan (2012) and assumed a spatially variable receiver fault plane for each calculation 


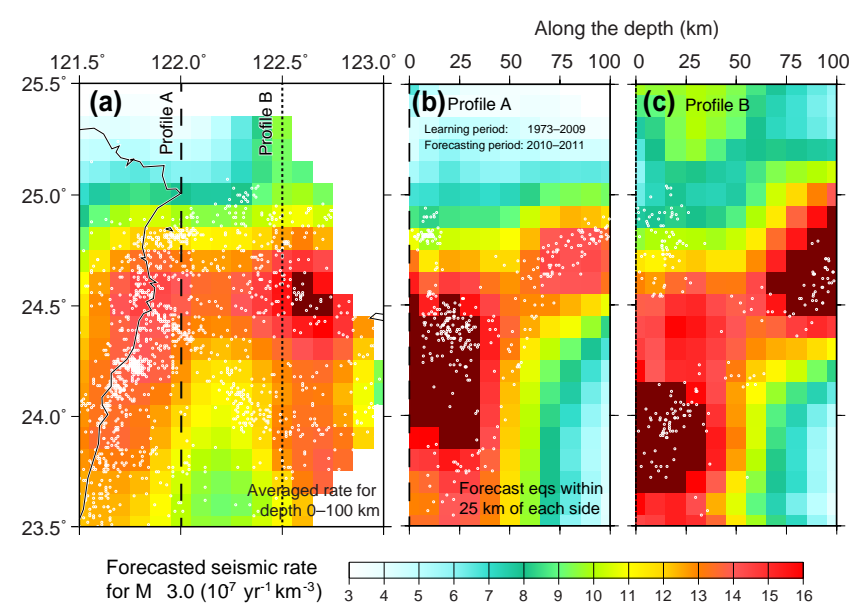

Figure 2. (a) A map view and (b-c) profiles of the forecasted seismicity rate for $M \geq 3.0$ modelled by the epicentre-smoothing kernel function. White dots denote the earthquakes from 2010 to 2011. Earthquakes within $25 \mathrm{~km}$ of each side of the profiles are presented.

grid. A receiver fault plane for each grid is consistent with the closest reference focal mechanism determined by $\mathrm{Wu}$ et al. (2010). For each grid node, I evaluated the $\triangle$ CFS on both nodal planes and reported the higher value. To minimize depth uncertainty, this study followed the procedure of Catalli and Chan (2012) that evaluated the $\triangle$ CFS for seismogenic depth and reported the maximum value for each calculation grid. Since earthquakes with small magnitudes do not significantly influence the current seismicity rate within the model (Catalli et al., 2008), I only analysed the $\Delta$ CFS for the $M \geq 4.5$ events (Table 1 ). The $\Delta$ CFS evaluation is based on the assumption of a homogeneous half space with an intermediate value of $\mu^{\prime}=0.4$, the Poisson's ratio of 0.24 and Young's modulus of $8 \times 10^{5}$ by applying the COULOMB 3.3 program (Toda and Stein, 2002). Application of the rate-andstate friction model (Eq. 5) requires parameters of $A \sigma$ and $t_{\mathrm{a}}$. Previous studies (e.g. Toda and Stein, 2003; Toda et al., 2005; Catalli et al., 2008) have suggested that the physically reasonable range for $A \sigma$ is between 0.1 and 0.4 bars. I assumed a fixed $A \sigma$ of 0.2 bars, corresponding to the assumption of previous studies (e.g. Chan et al., 2012, 2013). $t_{\mathrm{a}}$ was assumed to be a function of the moment magnitude $\left(M_{\mathrm{w}}\right)$, as proposed by Burkhard and Grünthal (2009), described as follows:

$t_{\mathrm{a}}=e^{\left(-4.77+\sqrt{0.62+17.32 \cdot M_{\mathrm{w}}}\right)}$ for $M_{\mathrm{w}}<7.8$,

$t_{\mathrm{a}}=e^{\left(6.44+0.06 \cdot M_{\mathrm{w}}\right)} \quad$ for $M_{\mathrm{w}} \geq 7.8$.

The unit of $t_{\mathrm{a}}$ is days. $t_{\mathrm{a}}$ is determined based on the magnitude of each source event (Table 1).

\subsection{Results}

\subsubsection{The two-dimensional models}

I first represent the forecasting models based on twodimensional calculation cells with a $0.1^{\circ} \times 0.1^{\circ}$ size (i.e. the depth-independent model). For application of the smoothing kernel function, $x-x_{i}$ in Eq. (1) was the epicentre distance between the site of interest and the epicentre of earthquakes (i.e. depth-independent). The model forecasted higher rates along the coastline of Taiwan and for the area east of latitude $122.5^{\circ}$, which correspond to the distribution of the forecasting events during 2010 and 2011 (Fig. 2a).

For the $\triangle$ CFS calculation on the two-dimensional grids, the target depth corresponds to the hypocentral depth of each source event (Table 1). Through the rate-and-state friction model, I calculated the time-dependent rate evolutions for different moments (Fig. 3). In comparison with the spatialtemporal pattern of the forecasting events (open circles in Fig. 3), many of the consequent earthquakes are in the region with rate decrease (green stars in Fig. 3); i.e. it is difficult to confirm the feasibility of this model.

\subsubsection{The three-dimensional models}

I then propose the forecasting models based on threedimensional cells with $0.1^{\circ} \times 0.1^{\circ} \times 10 \mathrm{~km}$ sizes (i.e. the depth-dependent model). For the smoothing kernel function application, $x-x_{i}$ in Eq. (1) was the distance between the site of interest and the hypocentre of earthquakes (i.e. depthdependent). Two profiles along the longitudes of 122.0 and $122.5^{\circ}$ (Fig. 2b and c, respectively) presented higher forecasted rates above the depth of $30 \mathrm{~km}$ and along the subduction slab dipping to the north, which fit the distribution of the forecasting earthquakes (the open circles in Fig. $2 b$ and c) well.

For the rate-and-state friction model application, I evaluated the maximum $\triangle \mathrm{CFS}$ along the seismogenic depth for each cell and modelled the corresponding seismicity rate evolution (Fig. 4). Departing from the outcomes of the twodimensional model (Fig. 3), a significant rate increase near the epicentre of each source event corresponds to the distribution of forecasting events (Fig. 4).

\section{Forecasting application to the Kanto region}

\subsection{Tectonic setting and earthquake catalogue}

The Kanto region, Japan, is an area with complex tectonic settings. Most parts of this region sit on the Eurasian Plate, under which the Philippine Sea Plate subducts from the south. At greater depth, the Pacific Plate subducts from the east (Toda et al., 2008). The complex plate interactions in this region result in seismic activity. Fortunately, similarly to the Ryukyu region, the activity has been well recorded by a 


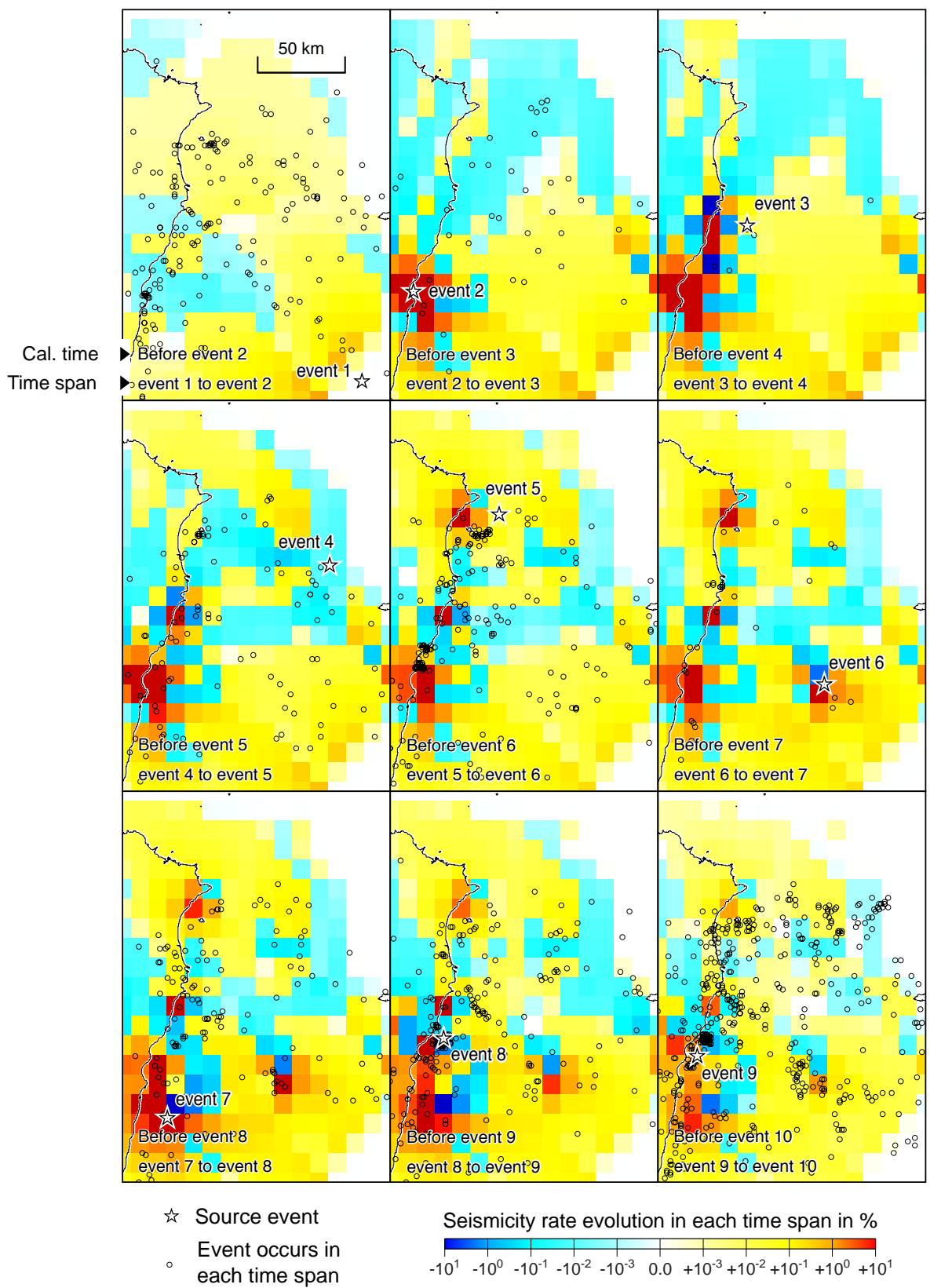

Figure 3. The seismicity rate evolution at different time moments. The target depth for the $\Delta$ CFS calculation corresponds to the hypocentral depth of each source event (Table 1). Source events from 2010 to 2011 are shown as open black stars. Open circles denote earthquakes during each time sequence.

high-quality seismic network. The modern seismic network maintained by the Japan Meteorological Agency (JMA) was initiated in 1923 and has been modernized over time (Nanjo et al., 2010). In addition, significant change in the seismicity behaviour in the Kanto region followed the 2011 M9.0 Tohoku earthquake (Ishibe et al., 2011; Toda et al., 2011). Such spatial-temporal conditions provide an ideal environment for testing the credibility of forecasting models in respect to both depth and time factors.

\subsection{Procedure of application}

Due to the seismic network modernization, $M_{\mathrm{c}}$ of the JMA catalogue decreased dramatically after 1980 and 1990 (Nanjo et al., 2010). Thus, I analysed the catalogue with various magnitude thresholds in the three periods: magnitudes 4.5, 3.5 and 2.5 since 1923, 1980 and 1990, respectively. The thresholds correspond to the $M_{\mathrm{c}}$ determined by Nanjo et al. (2010) through the entire magnitude range method (Woessner and Wiemer, 2005). I input the complete part of 


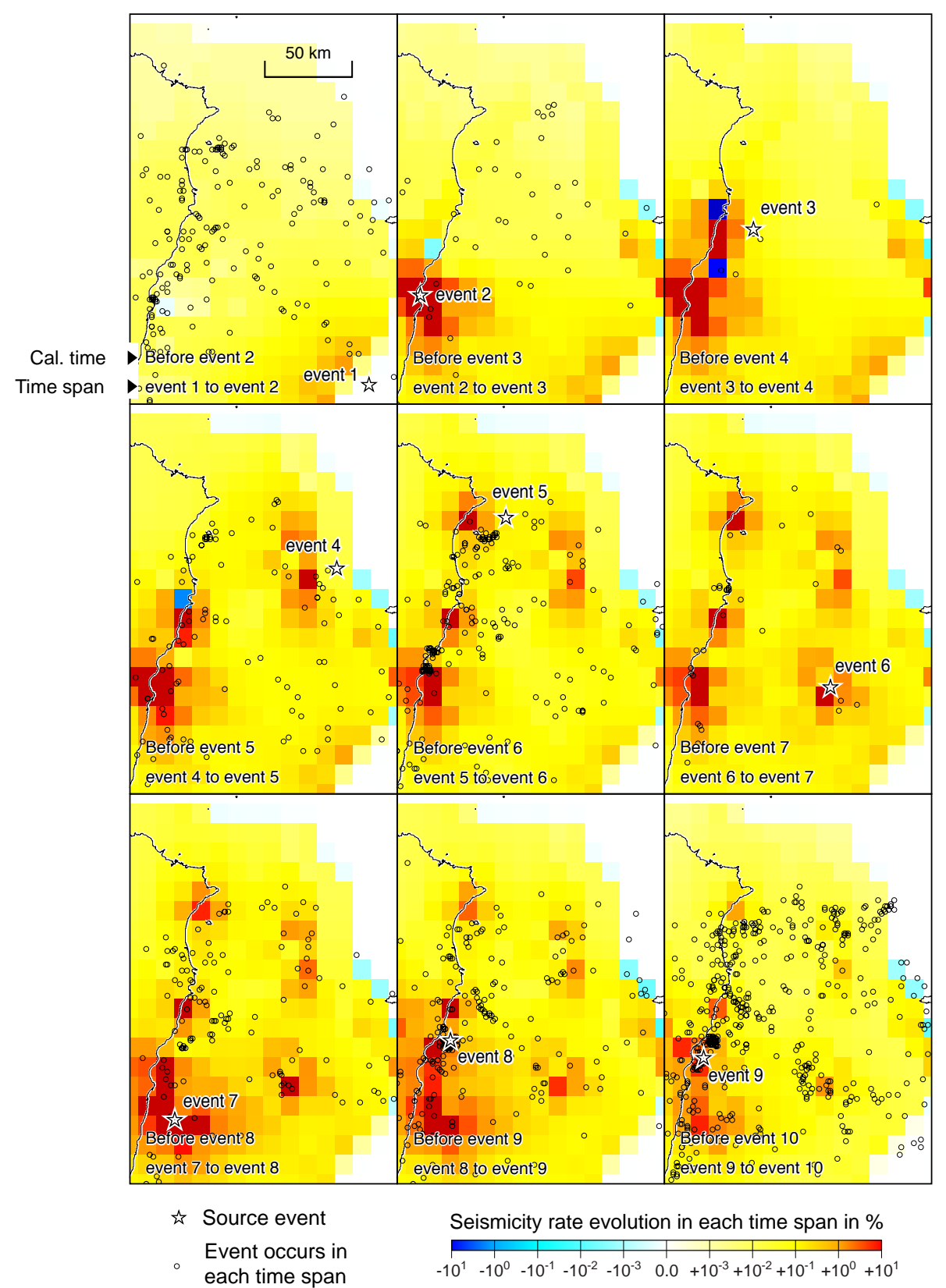

Figure 4. The seismicity rate evolution at different time moments. The $\triangle \mathrm{CFS}$ for each grid is defined as the maximum Coulomb stress changes for the entire seismogenic depth. Source parameters for the source events for calculating are shown in Table 1.

the JMA catalogue until the end of 2009 for forecast model construction, and referred those in 2010 and 2011 for retrospective tests. In this case, $T_{M}$ is 89,32 and 22 years, while $N_{\mathrm{M}}$ is the total number of earthquakes in the catalogue with magnitudes larger than $4.5,3.5$ and 2.5 , respectively. The linear regression determined that the $c$ and $d$ values of the bandwidth function were $0.9271 \mathrm{~km}$ and 0.6722 , respectively. The parameters obtained above provide a basis for the application of the smoothing kernel approach.
For the forecast using the rate-and-state friction model, I calculated the $\triangle \mathrm{CFS}$ of the $M \geq 6.0$ events during 2010 and 2011 (Fig. 5). The $\triangle$ CFS calculation for the 2011 M9.0 Tohoku earthquake is based on the coseismic dislocation model obtained by tsunami waveform inversion (Fujii et al., 2011). For the rest of the source events, I obtained the parameters from the F-net catalogue maintained by the National Research Institute for Earth Science and Disaster Prevention, Japan (NIED) (http://www.fnet.bosai.go.jp/ event/search.php?LANG=en), and determined fault dimen- 


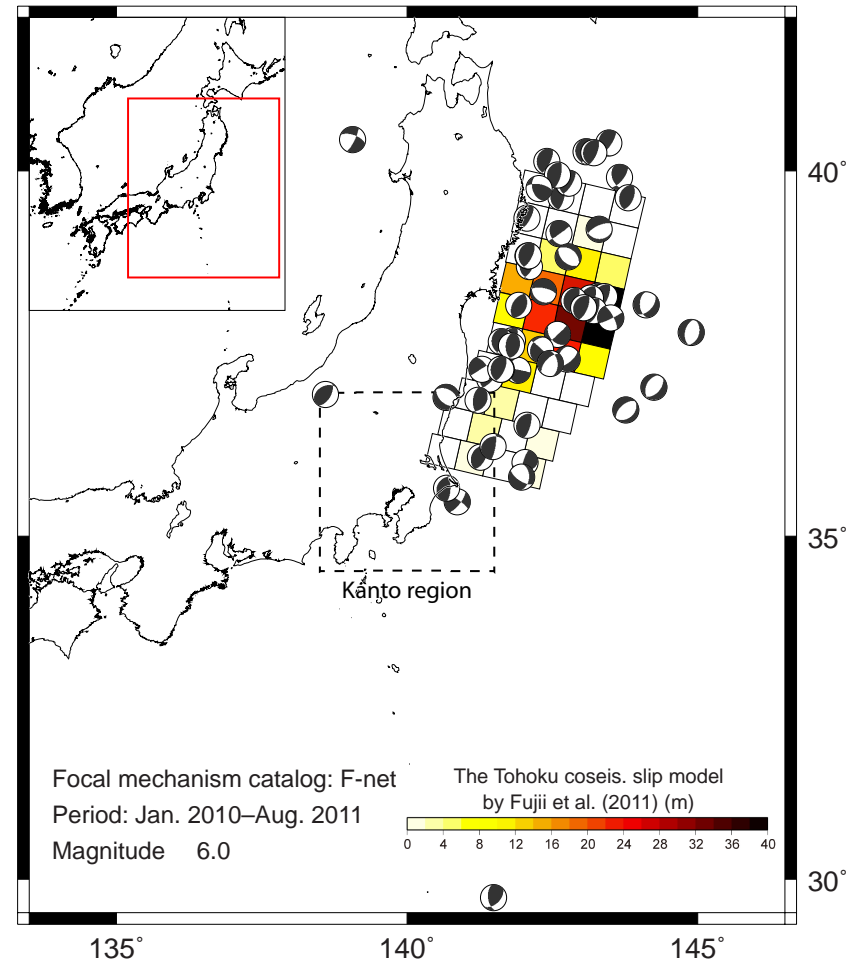

Figure 5. Distribution of the $M \geq 6.0$ earthquakes, which took place in or near the Kanto region during January of 2010 and August of 2011. The coseismic dislocation model of the 2011 M9.0 Tohoku earthquake is obtained by tsunami waveform inversion (Fujii et al., 2011), whereas the focal mechanisms of the others are obtained from the F-net catalogue.

sion and magnitude of slip through the scaling laws proposed by Wells and Coppersmith (1994). A receiver fault plane for each grid is consistent with the closest reference focal mechanism from the F-net catalogue.

\subsection{Results}

\subsubsection{The two-dimensional model}

This study first forecasts in two-dimensional calculation cells with $0.2^{\circ} \times 0.2^{\circ}$ sizes defined by the Collaboratory for the Study of Earthquake Predictability (CSEP) Japan Testing Center for the Kanto region (Tsuruoka et al., 2012). The target depth for the $\triangle \mathrm{CFS}$ calculation is $47.5 \mathrm{~km}$, which corresponds to the averaged hypocentral depth of the earthquakes in the region. Combining the smoothing kernel function and the rate-and-state friction model, the models represent higher seismicity rates for smaller magnitude ranges (e.g. Fig. 6a) than for larger ones (e.g. Fig. 6d), consistent with the Gutenberg-Richter law (Gutenberg and Richter, 1954). In addition, due to being stress-enhanced by the Tohoku sequence (Ishibe et al., 2011; Toda et al., 2011), a high seismicity rate is forecasted along the coast and offshore in the Pacific Ocean $40 \mathrm{~km}$ northeast of Tokyo. Note that the

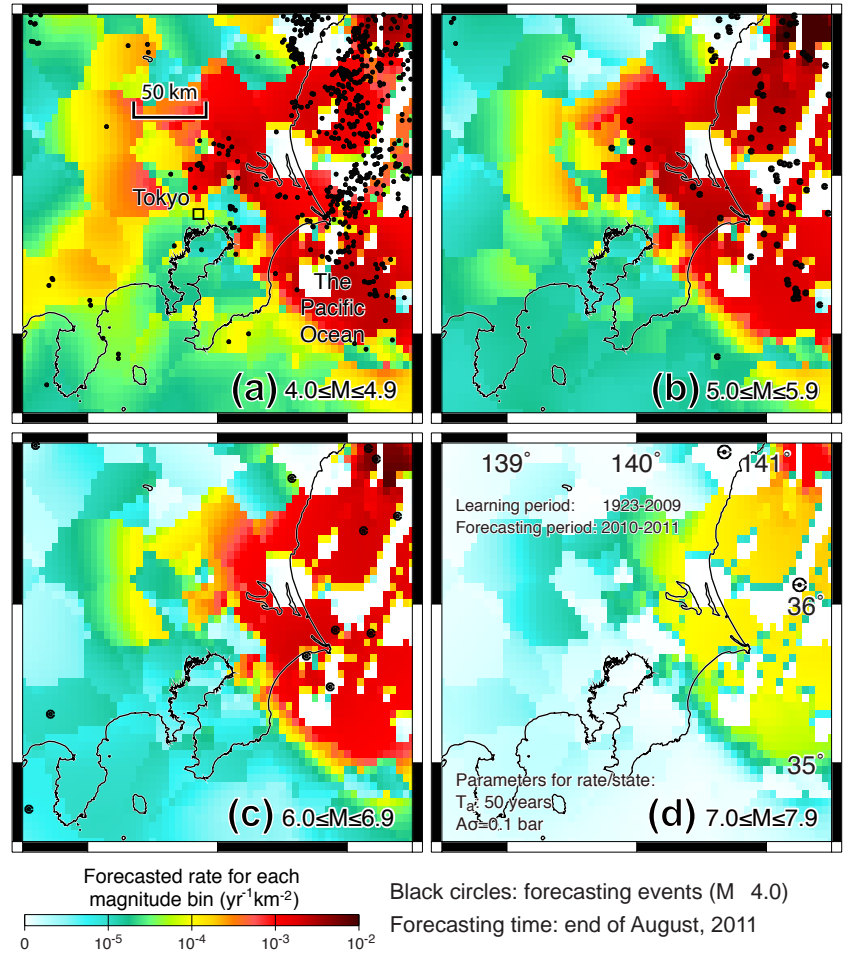

Figure 6. The two-dimensional forecasting models for the magnitudes between (a) 4.0 and 4.9; (b) 5.0 and 5.9; (c) 6.0 and 6.9; and (d) 7.0 and 7.9, respectively, at the end of August 2011. Black dots denote the $M \geq 4.0$ earthquakes during January 2010 and August 2011.

stress shadow zone at the target depth by the source events (including the 2011 Tohoku mainshock) resulted in some low forecasted rate zones offshore in the Pacific Ocean.

\subsubsection{The three-dimensional model}

I then proposed a depth-dependent model based on threedimensional cells with $0.2^{\circ} \times 0.2^{\circ} \times 5 \mathrm{~km}$ sizes. Compared with the forecast with two-dimensional cells, the threedimensional model illustrated more detailed patterns along the depth (Fig. 7b-d); e.g. the high seismicity rate $40 \mathrm{~km}$ northeast of Tokyo is identified at depths between 30 and $70 \mathrm{~km}$ (Fig. 7b), and the high rate along the coast and offshore in the Pacific Ocean is located at depths of $25-75 \mathrm{~km}$ (Fig. 7d), consistent with the boundary between the Pacific and Eurasia plates (Toda et al., 2008).

\section{Discussion and conclusion}

\subsection{Forecasting ability of each forecasting model}

To validate the forecasting ability statistically, I compared models with the distribution of forecasting earthquakes using the Molchan diagram (Molchan, 1990, and references 


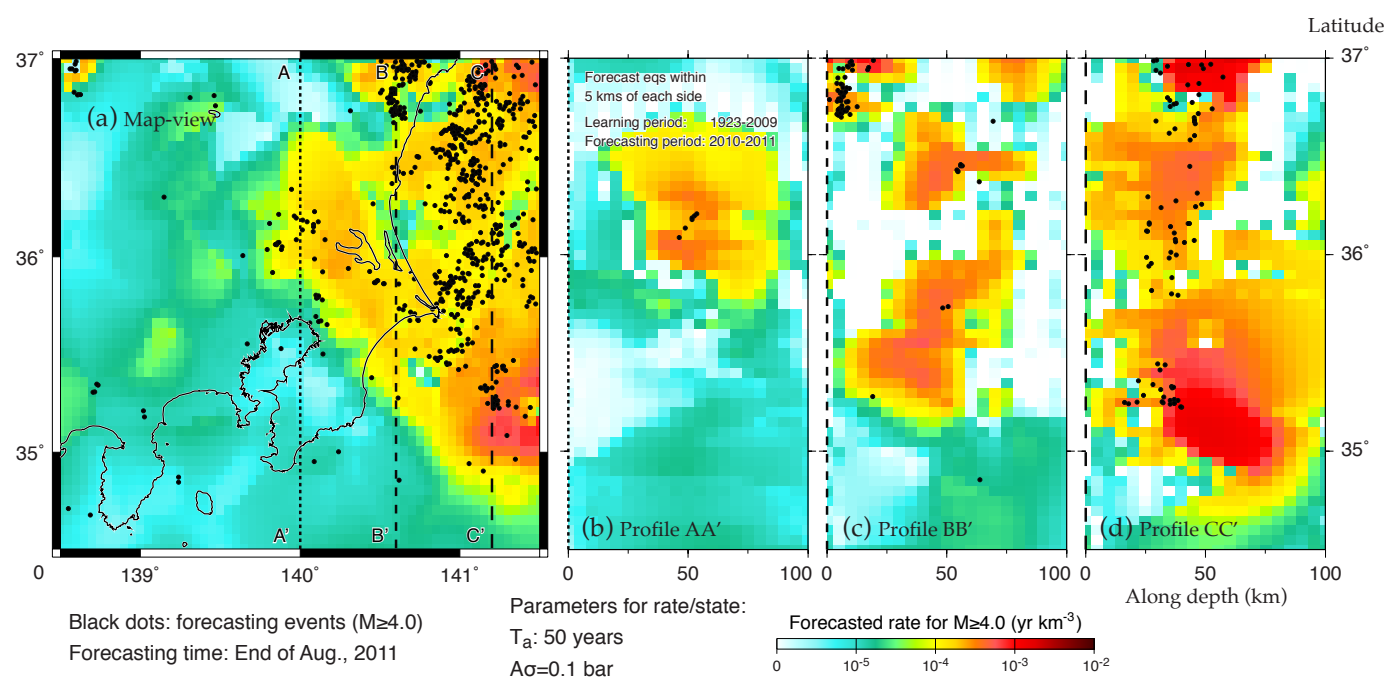

Figure 7. (a) Map view and (b)-(d) profiles of the three-dimensional forecasting models at the end of August 2011 and the distribution of the target earthquakes during 2010-2011. Black dots represent the $M \geq 4.0$ earthquakes during January 2010 and August 2011 . Earthquakes within $5 \mathrm{~km}$ of each side of the profiles are presented.

therein). The diagram was designed for evaluating forecasting ability through presenting the fraction of alarm-occupied space vs. the fraction of failure in forecasting by considering the locations of the forecasting earthquakes with respect to the distribution of forecasting seismicity density rate. The "fraction of alarm-occupied space" indicates the percentage of events within the study region with a forecasting level equal to or higher than "alarm". The "fraction of failure in forecasting" represents the percentage of consequent earthquakes with a lower forecasting level than the alarm, corresponding to "miss rate" defined by some previous studies (e.g. Zechar and Jordan, 2008). For each event, the area with a forecasting rate equal to or smaller than that at the location of the forecasting earthquake is extracted and represented as a percentage of the entire study area. The events are then sorted according to percentage of area and plotted against event count, represented as the percentage of the total number of forecasting events. In the diagram, when data points are distributed along a diagonal line, the distribution of target earthquakes is independent of forecasting; convex distribution suggests that the majority of consequent earthquakes occur within regions with a lower forecasted rate, whereas concavity suggests that the majority of consequent earthquakes are within a high forecasted rate area. An optimistic forecasting is represented in the Molchan diagram by the condition of having the lowest fraction of alarm-occupied space, and the lowest fraction of failure in forecasting.

Through Molchan diagrams, the forecasted seismicity rate obtained using different models was compared with the locations of earthquakes for the Ryukyu and Kanto cases (shown in Figs. 8 and 9, respectively). Most of the models show concavity distribution, suggesting good forecasting ability, except the case of the combining two models in

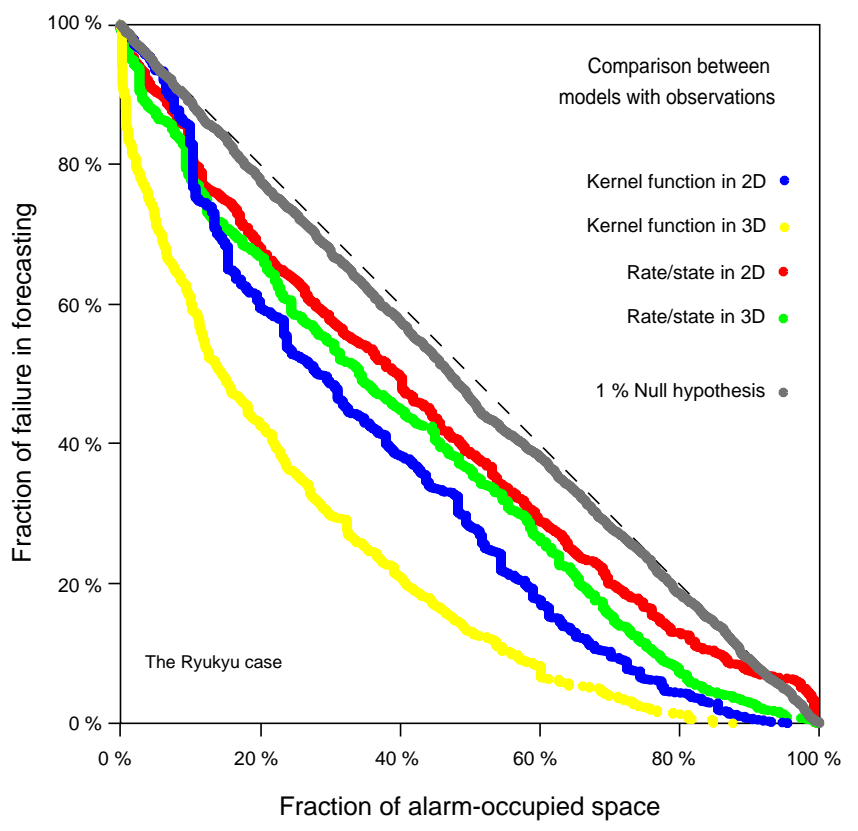

Figure 8. The Molchan diagram used for investigating the correlation between different forecasting models and earthquakes during the forecasting period (2010-2011) for the Ryukyu case. Blue and yellow dots represent the results for the models using the smoothing kernel models in two- and three-dimensional grids, respectively; red and green dots represent the results for the models using the rate-and-state friction models in two- and three-dimensional grids, respectively; grey dots represent the $99 \%$ significance level determined by 1640 forecasting events. 


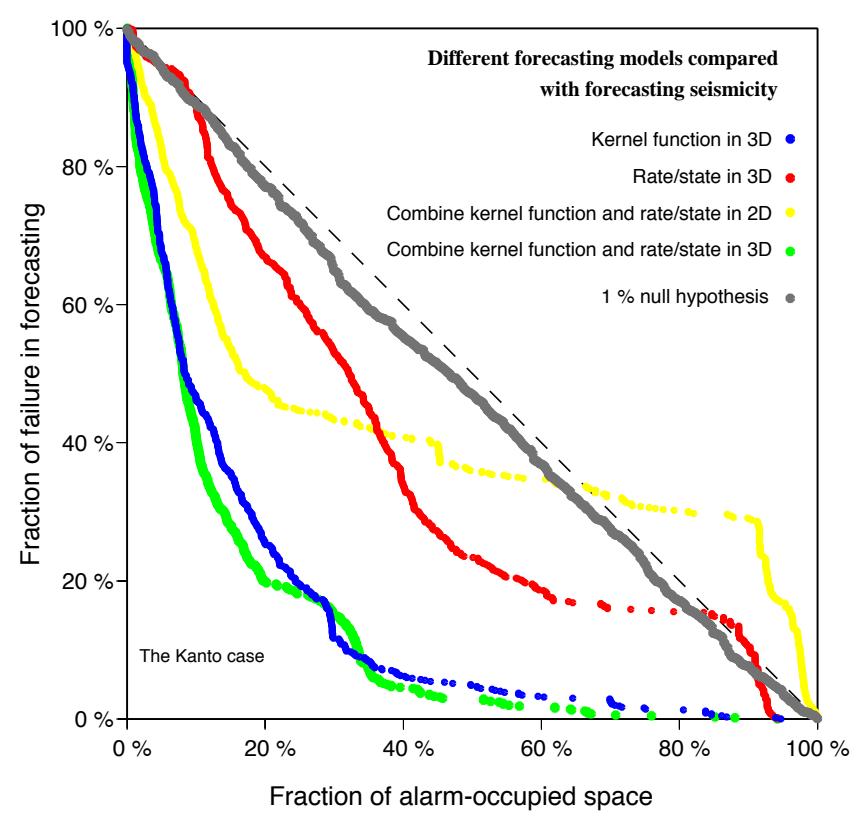

Figure 9. The Molchan diagram used for investigating the correlation between different forecasting models and earthquakes during the forecasting period (January 2010-August 2011) for the Kanto case. Blue and red dots denote the results for the models using solely the smoothing kernel function and the rate-and-state friction model, respectively; yellow and green dots denote the results for the combination of the two models in two- and three-dimensional grids, respectively; grey dots represent the $99 \%$ significance level determined by 703 forecasting events.

two-dimensional grids in Kanto (the yellow dots in Fig. 9). Such an exception can be attributed to slip model misfit and hypocentral depth uncertainties of forecasting earthquakes (Catalli and Chan, 2012). To further confirm the significance of the forecasting ability for the rest of models, the null hypothesis (Zechar and Jordan, 2008) is implemented. The $99 \%$ significance level, i.e. $\alpha=1 \%$ in Eq. (3) of Zechar and Jordan (2008) for both Ryukyu and Kanto cases (the grey dots in Figs. 8 and 9, respectively), is plotted based on the number of the forecasted earthquakes (1640 and 703 earthquakes, respectively). Most of the models cannot be rejected by the $99 \%$ confidence level of the null hypothesis (the data below the grey dots in Figs. 8 and 9), confirming their robustness.

\subsection{Importance of the temporal factor}

Since the smoothing kernel function averages the seismic activity during the observation period, it can be regarded as a time-independent model. In contrast, the rate-and-state friction model forecasts temporal evolution of seismicity rate disturbed by a series of source events and can be renewed with time. The comparison between the two models may indicate the importance of the temporal factor for forecasting.
The Molchan diagram in the Ryukyu case shows a lower fraction of failure in forecasting for the smoothing kernel model using the two-dimensional grids (the blue dots in Fig. 8) than that for the rate-and-state friction model (the red dots in Fig. 8) when fraction of alarm-occupied space is fixed. Such a result confirms a better forecasting ability for the smoothing kernel model. A similar conclusion can be obtained for the three-dimensional models (the yellow and green dotes in Fig. 8). This finding corresponds to the conclusion of Chan et al. (2012), obtained from forecasting experience in entire Taiwan. For the Kanto case (Fig. 9), by contrast, departing from conclusion of the Ryukyu case, the rate-and-state friction model provides a better performance.

The discrepant conclusions between the two cases might be attributed to the effect of recent earthquakes. For the cases of Ryukyu and Chan et al. (2012), there was no significant short-term rate perturbation during the forecasting periods. For the Kanto case, on the contrary, the time dependency becomes a crucial factor in forecasting the consequences of the 2011 Tohoku earthquake (Ishibe et al., 2011; Toda et al., 2011).

\subsection{Importance of the depth factor}

Both of the Ryukyu and Kanto cases have qualitatively shown that three-dimensional models have a better performance in Figs. 2 and 7, respectively. The smoothing kernel function using three-dimensional grids forecasted distribution along depth in detail. For the rate-and-state friction model, the three-dimensional applications presented significant rate increase for most regions near the epicentre of each source event, corresponding to the locations of forecasting events.

For the Ryukyu application, comparing between forecasted rates obtained using the smoothing kernel function and the locations of target earthquakes using the Molchan diagram (blue and yellow dots in Fig. 8), the three-dimensional forecasting model had a better forecasting ability, i.e. a smaller fraction of failure to predict. For the rate-and-state friction model, the three-dimensional applications grids also provide a better forecasting ability (green dots in Fig. 8) in comparison to the two-dimensional ones (red dots in Fig. 8).

The application to the Kanto region also confirmed the better performance of the three-dimensional models (yellow and green dots in Fig. 9). In addition, in the Kanto case, the Molchan diagram raised the disadvantage for the rate-andstate model using two-dimensional calculation grids. When the fraction of alarm-occupied space is large, convex distributions are presented (red and yellow dots in Fig. 9); i.e. some earthquakes took place in the region with low/no forecasted rates (region in white in Fig. 6), suggesting forecasting failure. In contrast to the two-dimensional models, the threedimensional ones have proved their forecasting ability (green dots in Fig. 9). The conclusion is consistent with the findings of Catalli and Chan (2012), and confirms that the depth fac- 
tor is one of the upmost important parameters for Coulomb stress calculation.

Through the applications to different forecasting approaches, I have confirmed that models with threedimensional grids always obtain better forecasting ability. In addition, the importance of depth dependency for forecasting models is determined, especially for the application to a subduction environment or to a region with non-vertical seismogenic structures.

\section{Data availability}

In the Ryukyu case, the earthquake parameters were obtained from the catalogue of the Central Weather Bureau Seismic Network (last accessed December 2013); the focal mechanisms parameters were obtained from Broadband Array in Taiwan for Seismology (BATS) (last accessed December 2013). In the Kanto case, the earthquake parameters were obtained from the catalogue of the Japan Meteorological Agency (JMA) network (last accessed April 2012); the focal mechanisms parameters were obtained from the F-net catalogue (last accessed April 2012). The detailed slip dislocation model of the 2011 M9.0 Tohoku earthquake was derived from Fujii et al. (2011) (http://iisee.kenken.go. jp/staff/fujii/OffTohokuPacific2011/tsunami_inv.html). The Coulomb stress change is calculated from COULOMB 3.3 (http://usgsprojects.org/coulomb/).

Acknowledgements. The work was supported by the Earthquake Research Institute, the University of Tokyo and the Earth Observatory of Singapore, Nanyang Technological University. The author would like to thank CWB, BATS, JMA and NIED for providing the earthquake catalogues used in this study, and Stefano Tinti and two anonymous reviewers for their constructive comments.

Edited by: S. Tinti

Reviewed by: two anonymous referees

\section{References}

Beauval, C., Scotti, O., and Bonilla, F.: The role of seismicity models in probabilistic seismic hazard estimation: comparison of a zoning and a smoothing approach, Geophys. J. Int., 165, 584595, 2006.

Burkhard, M. and Grünthal, G.: Seismic source zone characterization for the seismic hazard assessment project PEGASOS by the Expert Group 2 (EG 1b), Swiss J. Geosci., 102, 149-188, 2009.

Catalli, F. and Chan, C. H.: New insights into the application of the Coulomb model in real-time, Geophys. J. Int., 188, 583-599, doi:10.1111/j.1365-246X.2011.05276.x, 2012.

Catalli, F., Cocco, M., Console, R., and Chiaraluce, L.: Modeling seismicity rate changes during the 1997 Umbria-Marche sequence (central Italy) through a rate- and state-dependent model, J. Geophys. Res., 113, B11301, doi:10.1029/2007JB005356, 2008.
Chan, C. H., Sørensen, M. B., Stromeyer, D., Grünthal, G., Heidbach, O., Hakimhashemi, A., and Catalli, F.: Forecasting Italian seismicity through a spatio-temporal physical model: importance of considering time dependency and reliability of the forecast, Ann. Geophys. Italy, 53, 129-140, doi:10.4401/ag-4761, 2010.

Chan, C.-H., Wu, Y.-M., and Wang, J.-P.: Earthquake forecasting using the rate-and-state friction model and a smoothing Kernel: application to Taiwan, Nat. Hazards Earth Syst. Sci., 12, 30453057, doi:10.5194/nhess-12-3045-2012, 2012.

Chan, C.-H., Wu, Y.-M., Cheng, C.-T., Lin, P.-S., and Wu, Y.-C.: Time-dependent probabilistic seismic hazard assessment and its application to Hualien City, Taiwan, Nat. Hazards Earth Syst. Sci., 13, 1143-1158, doi:10.5194/nhess-13-1143-2013, 2013.

Cocco, M. and Rice, J. R.: Pore pressure and poroelasticity effects in Coulomb stress analysis of earthquake interactions, J. Geophys. Res., 107, 2030, doi:10.1029/2000JB000138, 2002.

Dieterich, J. H.: A constitutive law for rate of earthquake production and its application to earthquake clustering, J. Geophys. Res., 99, 2601-2618, 1994.

Fujii, Y., Satake, K., Sakai, S., Shinohara, M., and Kanazawa, T.: Tsunami source of the 2011 off the Pacific coast of Tohoku Earthquake, Earth Planets Space, 63, 815-820, 2011.

Gutenberg, B. and Richter, C.: Seismicity of the Earth and Associated Phenomena, 2nd ed., 310 pp., Princeton Univ. Press, Princeton, NJ, 1954.

Harris, R. A.: Introduction to special section, Stress triggers, stress shadows, and implications for seismic hazard, J. Geophys. Res., 103, 24347-24358, 1998.

Ishibe, T., Shimazaki, K., Satake, K., and Tsuruoka, H.: Change in seismicity beneath the Tokyo metropolitan area due to the 2011 off the Pacific coast of Tohoku earthquake, Earth Planets Space, 63, 731-735, doi:10.5047/eps.2011.06.001, 2011.

Lombardi, A. M. and Marzocchi, W.: Double Branching model to forecast the next $M \geq 5.5$ earthquakes in Italy, Tectonophysics, 475, 514-523, 2009.

Marzocchi, W., Sandri, L., and Boschi, E.: On the validation of earthquake-forecasting models: the case of pattern recognition algorithms, Bull. Seismol. Soc. Am., 93, 1994-2004, 2003.

Molchan, G. M.: Strategies in strong earthquake prediction, Phys. Earth Planet. Int., 61, 84-98, 1990.

Molina, S., Lindholm, C. D., and Bungum, H.: Probabilistic seismic hazard analysis: zoning free versus zoning methodology, B. Geofis. Teor. Appl., 42, 19-39, 2001.

Nanjo, K. Z., Ishibe, T., Tsuruoka, H., Schorlemmer, D., Ishigaki, Y., and Hirata, N.: Analysis of the completeness magnitude and seismic net- work coverage of Japan, Bull. Seismol. Soc. Am., 100, 3261-3268, doi:10.1785/0120100077, 2010.

Shin, T. C.: Some implications of Taiwan tectonic features from the data collected by the Central Weather Bureau Seismic Network, Meteorol. Bull., 38, 23-48, 1992 (in Chinese).

Toda, S. and Stein, R. S.: Response of the San Andreas Fault to the 1983 Coalinga-Nuñez Earthquakes: An Application of Interaction-based Probabilities for Parkfield, J. Geophys. Res., 107, ESE 6-1-ESE 6-16, doi:10.1029/2001JB000172, 2002.

Toda, S. and Stein, R. S.: Toggling of seismicity by the 1997 Kagoshima earthquake couplet: A demonstration of time-dependent stress transfer, J. Geophys. Res., 108, 2567, doi:10.1029/2003JB002527, 2003. 
Toda, S., Stein, R. S., Richards-Dinger, K., and Bozkurt, S. B.: Forecasting the evolution of seismicity in southern California: Animations built on earthquake stress transfer, J. Geophys. Res., 110, B05S16, doi:10.1029/2004JB003415, 2005.

Toda, S., Stein, R. S., Kirby, S. H., and Bozkurt, S. B.: A slab fragment wedged under Tokyo and its tectonic and seismic implications, Nat. Geosci., 1, 1-6, doi:10.1038/ngeo1318, 2008.

Toda, S., Stein, R. S., and Lin, J.: Widespread seismicity excitation throughout central Japan following the $2011 \mathrm{M}=9.0$ Tohoku earthquake and its interpretation by Coulomb stress transfer, Geophys. Res. Lett., 38, L00G03, doi:10.1029/2011GL047834, 2011.

Tsai, Y. B., Liaw, Z. S., and Lee, T. Q.: A statistical study of the Taiwan Telemetered Seismographic Network data during 19731979, Bull. Inst. Earth Sci. Acad. Sin., 1, 1-22, 1981.

Wells, D. L. and Coppersmith, K. J.: New empirical relationships among magnitude, rupture length, rupture width, rupture area, and surface displacement, Bull. Seismol. Soc. Am., 84, 9741002, 1994.

Wiemer, S. and Wyss, M.: Minimum Magnitude of Completeness in Earthquake Catalogs: Examples from Alaska, the Western United States, and Japan, Bull. Seismol. Soc. Am., 90, 859-869, 2000.
Wiens, D. A., Gilbert, H. J., Hicks, B., Wysession, M. E., and Shore, P. J.: Aftershock sequences of moderate-sized intermediate and deep earthquakes in the Tonga Subduction Zone, Geophys. Res. Lett., 24, 2059-2062, 1997.

Woessner, J. and Wiemer: S., Assessing the quality of earthquake catalogues: Estimating the magnitude of completeness and its uncertainty, Bull. Seismol. Soc. Am., 95, 684-698, 2005.

Woo, G.: Kernel Estimation Methods for Seismic Hazard Area Source Modeling, Bull. Seismol. Soc. Am., 86, 353-362, 1996.

Wu, Y. M., Hsu, Y. J., Chang, C. H., Teng, L. S., and Nakamura, M.: Temporal and spatial variation of stress field in Taiwan from 1991 to 2007: Insights from comprehensive first motion focal mechanism catalog, Earth Planet. Sci. Lett., 298, 306-316, doi:10.1016/j.eps1.2010.07.047, 2010.

Yen, Y. T. and Ma, K. F.: Source-Scaling Relationship for M4.68.9 Earthquakes, Specifically for Earthquakes in the Collision Zone of Taiwan, Bull. Seismol. Soc. Am., 101, , 464-481, doi:10.1785/0120100046, 2011.

Zechar, J. D. and Jordan, T. H.: Testing alarm-based earthquake predictions, Geophys. J. Int., 172, 715-724, 2008. 